\title{
Anti-adhesive Effect of Hyaluronate in a Rabbit Laminectomy Model
}

\author{
Jian-Ming Chen ${ }^{1}$, Sheng-Hsun Lee ${ }^{1}$, Tsung-Ting Tsai ${ }^{1,2}$, Chi-Chien Niu ${ }^{1}$, Lih-Huei Chen ${ }^{1}$, \\ Wen-Jer Chen ${ }^{1}$
}

Background: Postlaminectomy dural adhesion is a common cause of recurrent symptoms. Hyaluronic acid-based gel has been reported to reduce the incidence of postoperative adhesion in the peritoneal cavity; however, its effect on preventing postoperative scar formation at laminectomy sites is not yet known. The purpose of this study was to evaluate the anti-adhesive effect of hyaluronic acid-based gelatin after laminectomy, using a rabbit model.

Methods: Twelve adult New Zealand rabbits underwent two-level lumbar laminectomy, and were randomly assigned to one of two groups of six rabbits each. The treatment group received hyaluronic acid-based gelatin treatment and the control group was untreated. Rabbits were sacrificed 8 weeks after treatment. Peel-off testing and histological analysis were performed to assess the tenacity and the extent of adhesion formation.

Results: $\quad$ No significant difference was observed in the neurologic performance between the two groups. The tenacity in the treatment group was significantly reduced compared to that of the control group $(3.17 \pm 0.75$ vs. $4.33 \pm 0.52$, respectively; $p=0.016)$. Histological analysis showed significantly less scar tissue formation in the treatment group, with a larger subarachnoid space and greater distance between the dura and scar tissues. The amount of fibroblast cells also was significantly smaller in the treatment group than in the control group (3078 \pm 313.68 vs. $3742 \pm 455.65$, respectively; $p=0.042$ ).

Conclusions: No serious adverse events were reported, and no difference was found in the incidence of complications between the treatment and control groups. The findings suggested that hyaluronic acid-based gelatin may be effective for preventing postlaminectomy dural adhesion in rabbits.

(Biomed J 2014;37:218-224)

Key words: adhesives, hyaluronate, laminectomy, rabbits, scarring

$\mathrm{P}$ ostoperative fibrosis after spinal surgeries such as discectomy and laminectomy can sometimes cause neurologic side effects due to direct compression of the

\begin{abstract}
At a Glance Commentary
Scientific background of the subject

Hyaluronic acid has been widely used in numerous clinical applications. It has been used to treat knee osteoarthritis by injecting it into the joint. It can act as an anti-adhesion barrier in preventing postoperative scar tissue formation in various surgical procedures such as myomectomy and laparotomy.
\end{abstract}

\section{What this study adds to the field}

Hyaluronic acid-based gel is effective in decreasing the tenacity of adhesion between the dura and scar and the amount of scar tissues after laminectomy in a rabbit model, which can be considered as a potential anti-adhesion barrier in preventing postoperative adhesion formation.

\footnotetext{
J. M. Chen and S. H. Lee contributed equally to this article.

From the ${ }^{1}$ Department of Orthopaedic Surgery, Spine Section, Chang Gung Memorial Hospital at Linkou, Chang Gung University College of Medicine, Taoyuan, Taiwan; ${ }^{2}$ Graduate Institute of Clinical Medical Sciences, College of Medicine, Chang Gung University, Taoyuan, Taiwan

Received: Mar. 30, 2013; Accepted: Aug. 05, 2013

Correspondence to: Dr. Tsung-Ting Tsai, Department of Orthopaedic Surgery, Spine Section, Chang Gung Memorial Hospital at Linkou. 5 , Fusing St., Gueishan, Taoyuan 333, Taiwan (R.O.C). Tel: 886-3-3281200 ext. 3612; Fax: 886-3-3278113; E-mail: tsai1129@ gmail.com
}

DOI: $10.4103 / 2319-4170.117894$ 
relationship between postoperative fibrosis and recurrent radiculopathy or low back pain after a successful discectomy. However, there is no well-defined evidence to support the fact that postoperative fibrosis is the major cause of physical impairment when no bony or other pathologic tissue was detected during the revision surgery. ${ }^{[1,2]}$ Although some patients may consider undergoing subsequent surgeries for relief of their intolerable symptoms, the outcome is often unpredictable and the rate of complications such as iatrogenic nerve root injury or dural tear is relatively high. It is difficult for spine surgeons to confidently predict the benefits of such surgeries, which may also lead to legal problems. In addition, due to further degeneration at the surgical level or in the nearby areas, postoperative fibrosis can become more complicated and require longer surgeries, causing increased bleeding and risks associated with anesthesia, especially for elderly patients. ${ }^{[3,4]}$

Controlling fibrosis formation has been an important issue in successful spinal surgery. Many materials reportedly have the potential to prevent postoperative peridural adhesion or fibrosis, including physical materials such as barrier gels, Silastic ${ }^{\circledR}$ sheets (Dow Corning Corp., Midland, MI, USA), and absorbable foams, chemical materials such as nonsteroid anti-inflammatory drugs (NSAIDs), pharmaceuticals, and steroids, and biologic materials such as hyaluronate membranes, cellulose mesh, and free and pedicle fat grafts. ${ }^{[5-10]}$ However, the effects of these methods and materials remain questionable due to several extraneous and confounding variables in the experimental designs and various anti-adhesive agents.

The theory of fibrosis formation has been well studied and is described as growth of the fibrous connective tissue into the surrounding hematoma after laminectomy. To prevent hematoma infiltration, the anti-adhesive materials must fill the space, thus providing a tamponade effect, preventing hematomatous material from entering the epidural space. Hyaluronic acid (HA) is one of the anti-adhesive materials that have a predicable half-life, stable quality, and elastic configuration. In addition, its biocompatibility and efficacy in the prevention of postoperative adhesions in general surgery or gynecologic procedures have been studied for many years. Also, it has been widely used in the treatment of degenerative knee joint disease and cosmetics, and poses no hazards to patients. ${ }^{[11,12]}$ However, HA's effect on the prevention of postlaminectomy scar formation has not yet been tested. The purpose of our study was to examine the effects of HA on the prevention of postlaminectomy fibrosis formation and adhesion, using a rabbit model and histological analysis, gross observation, and peel-off testing.

\section{METHODS}

\section{Materials and animals}

The HA-based gelatin that was used in this study was Synvisc $^{\circledR}$ (hylan G-F 20; Genzyme Biosurgery Corp., Ridgefield, NJ, USA). It is a mixture containing hylan A fluid, hylan B gel, and saltwater, with a high molecular weight of $6000 \mathrm{kDa}$. Hylans are made from sodium hyaluronate.

Animals for the experiment were obtained from the Laboratory Animal Center, Chang Gung Memorial Hospital. The principles and procedures of animal care and use were followed and approved by the Institutional Animal Care and Use committees of Chang Gung Memorial Hospital. A total of 12 adult New Zealand rabbits (weighing 2.8-3.2 kg each) were randomly divided into a treatment and a control group, each with 6 rabbits. A two-level lumbar laminectomy was performed in all rabbits. The treatment group was then treated with HA-based gelatin and the control group was untreated.

\section{Surgical procedure}

The surgical procedure was similar to that of human spinal laminectomy. Briefly, after general anesthesia, the surgical site was shaved and prepared. A $3 \mathrm{~cm}$ posterior longitudinal incision was made at the level of the fourth and fifth lumbar vertebrae, using meticulous hemostasis. Then, the soft tissue was dissected subperiosteally. The lumbar vertebral segments were exposed, and a total laminectomy was performed at L4-L5, using a power burr and a lamina punch, leaving the dura mater intact [Figure $1 \mathrm{~A}$ and $\mathrm{B}$ ].

For the treatment group, the dural theca was exposed after the laminectomy and the topical HA (Synvisc) was applied, so that it entirely covered the exposed spinal cord and roots [Figure 1C]. In contrast, the control group received no additional procedure. The fascia layer was closed with 2-0 VICRYL ${ }^{\mathrm{TM}}$ suture (Ethicon, Inc., Blue Ash, OH, USA) and then the skin was closed with 3-0 nylon suture. No iatrogenic dural or root lesions and no postoperative infection and neurologic deficit were noted. All rabbits were sacrificed 8 weeks after laminectomy.

\section{Gross observation and peel-off testing}

After euthanasia, blunt dissection was performed at the laminectomy site, and the residual lamina and pedicle containing the dural tube were all removed, exposing the spinal cord and surrounding scar tissue. The amount of scar tissue was graded by gross observation and scored on a scale from 1 to 3, where 1 ("mild") indicated scarring that affected less than $25 \%$ of the surgical field, 2 ("medium") indicated scarring that affected about $25-50 \%$ of the surgical field, 


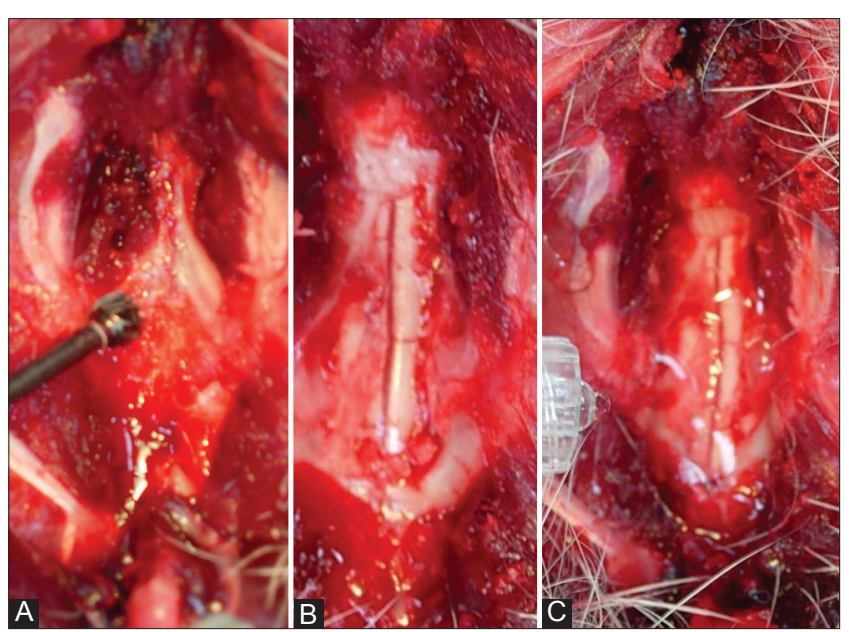

Figure 1: Surgical procedure. (A and B) A L4-L5 total laminectomy was performed with a power burr and a lamina punch, so that the dura mater remained intact. (C) Topical HA was immersed slowly to entirely cover the exposed spinal cord and roots.

and 3 ("large") was given where scarring was seen in more than $50 \%$ of the surgical field. ${ }^{[11]}$

Using forceps, the scar tissue was manually peeled off; the tenacity of adhesion between the scar tissue and dura was evaluated, and the difficulty of reopening the operated site was also taken into consideration. A six-level scoring system (grades 0-5) was used to indicate the difficulty of reopening the surgical site. With Grade 0, there was no adhesion between the dura and scar tissue. Grade 1 indicated very slight adhesion to the dura mater, and the tissue could be easily detached without applying manual force. With Grade 2, there was some adhesion to the dura and the tissue could be easily detached by moderate traction. With Grade 3, less than $50 \%$ of the operated area had adhesion and could be detached by strong traction. In Grade 4, more than $50 \%$ of the operated area had adhesion and could be bluntly detached by strong traction. Grade 5 indicated severe adhesion that could not be detached without disruption of the dura mater, unless sharp dissection was used. ${ }^{[12,13]}$

The grading systems of gross observation and peel-off testing were based on a random selection of rabbits. The assigned group and treatment status were revealed to the observer only after the scoring was done.

\section{Histological examination}

The tissue samples at the laminectomy site were harvested after the rabbits were sacrificed. The samples were fixed in formaldehyde solution, immersed into ethylenediaminetetraacetic acid (EDTA) for decalcification, and embedded in paraffin. The sections were deparaffinized in xylene and rehydrated through graded ethanol washes, followed by staining with standard hematoxylin and eosin. All sections were evaluated by the same histologist, who was blinded to the group and study method. The extent of adhesion (dura/root involvement score) was graded according to the method of He et al. ${ }^{[6]}$ In Grade 0 adhesions, the dura mater was free of scar tissue. In Grade 1 adhesions, only thin fibrosis bands between the scar tissues and the dura mater were observed. In Grade 2 adhesions, continuous adhesion was observed but made up less than two-thirds of the laminectomy defect. In Grade 3 adhesions, scar adhesion was large and involved more than two-thirds of the laminectomy defect, and/or extended to the nerve roots.

The scar tissue consistency and inflammatory response were graded using the scoring system proposed by Miyamoto et al ${ }^{[8]}$ These researchers used a 4-point scoring system. A score of 0 indicated loose connective tissue with small collagen bundles, the presence of highly vascular adipose tissue, with moderate macrophage and inflammatory cell activity. A score of 1 indicated connective tissue density, edges of defect with evidence of new bone formation, and mild macrophage and inflammatory cell activity. A score of 2 was used for dense connective tissue and/or fibrocartilage, absence of adipose tissue, avascular tissue, and absence of macrophage and inflammatory cell activity. A score of 3 indicated dense collagenous connective tissue, absence of adipose tissue, avascular tissue, and absence of macrophage and inflammatory cell activity. In addition, fibroblasts were counted ( $\times 400$ magnification), and the results from the two groups were compared.

\section{Statistical analysis}

Mann-Whitney U test was used to compare the values of each parameter between the control and treatment groups. Numerical data were presented as the mean plus or minus the standard deviation (Mean \pm SD), while categorical data were expressed as absolute frequencies using SPSS software. A $p<0.05$ was considered statistically significant.

\section{RESULTS}

\section{Surgical outcome}

All 12 rabbits were smoothly ambulatory after surgery without obvious neurologic deficits. The fascia, muscle, and skin around the surgical levels healed well in both groups, and no infection or discharge from the wound was noted at sacrifice.

\section{Gross observation and peel-off testing}

Gross observation showed that scar tissue occurred in the center of the laminectomy defect, and no residue of HA was found in either group, 8 weeks after laminectomy. In the control group, all scored 3 because a large amount of scar tissue formation was found in the laminectomy site. In 
the treatment group, three scored 3 and the remaining three scored 2 for medium scar tissue formation. Wounds treated with HA showed a relatively smaller amount of scar tissue in comparison to untreated wounds $(p=0.056)$. Comparison of adhesion in both groups is shown in Table 1.

In manual peel-off testing, the mean of the scores was $4.33 \pm 0.52$ for the control group and $3.17 \pm 0.75$ for the treatment group. There was a significant difference in the tenacity of adhesion between the scar tissue and dura in the two groups $(p=0.016)$.

\section{Histological analysis}

The mean of the "dura/root involvement score" for the extent of scar adhesion was $2.00 \pm 0.63$ in both groups. However, the score for the scar tissue consistency and inflammatory response was slightly lower in the treatment group, with a mean of $1.67 \pm 0.52$, whereas the mean was $2.33 \pm 0.52$ in the control group $(p=0.056)$.

Furthermore, the histological results showed that the harvested tissue samples covered with HA seemed to have a larger subarachnoid space, a greater distance from the surface of the dura to scar tissue, and a smaller number of inflammatory cells in the scar tissue at the laminectomy site [Figure 2].

The density of fibroblast cells was significantly less in the treatment group with a mean of $3078 \pm 313.68$ cells than in the control group with a mean of $3742 \pm 455.65$ cells $(p=0.041)$.

\section{DISCUSSION}

Postoperative fibrosis and adhesion are normal components of the healing process. Fibrosis and adhesion are the result of an inflammatory reaction caused by the organization of the fibrin matrix, and these steps are necessary in tissue healing. ${ }^{[14]}$ However, in cases of marked postoperative peridural fibrosis, fibrosis and adhesion can cause tethering of nerve roots and subsequent neurologic symptoms. It is also known that peridural scar adhesion is one of the causes of persistent radiculopathy. ${ }^{[4]}$ In a randomized prospective study, Ross et al. ${ }^{[2]}$ demonstrated a strong correlation between scar adhesion and postoperative pain. Patients with

Table 1: Comparative results of analyzing adhesion between the two groups

\begin{tabular}{lccccccccc}
\hline & \multicolumn{3}{c}{ Control group } & & \multicolumn{4}{c}{ Treatment group } \\
\cline { 2 - 4 } \cline { 7 - 9 } & Mean & SD & Median & & Mean & SD & Median & $p$ value \\
\hline Scar tissue amount & 3.00 & 0.00 & 3 & & 2.50 & 0.55 & 3 & 0.056 \\
Scar tissue tenacity & 4.33 & 0.52 & 4 & & 3.17 & 0.75 & 3 & 0.016 \\
$\begin{array}{l}\text { Dura/root } \\
\text { involvement score }\end{array}$ & 2.00 & 0.63 & 2 & & 2.00 & 0.63 & 2 & 1.000 \\
$\begin{array}{l}\text { Scar tissue } \\
\text { consistency }\end{array}$ & 2.33 & 0.52 & 2 & & 1.67 & 0.52 & 2 & 0.056 \\
\hline
\end{tabular}

extensive peridural scar adhesion were 3.2 times more likely to experience recurrent radicular pain than the patients with less extensive scarring. In addition to persistent postoperative symptoms, peridural fibrosis may also pose greater risks, including iatrogenic dura injury and nerve root injury; they often have more difficulties during surgery, longer operation times, and more bleeding when revision surgery is needed.

There have been several mechanisms proposed to explain the presence of postlaminectomy dural adhesion. While Key et al., ${ }^{[15]}$ suggested that epidural fibrosis may come from the annulus fibrosis of the disc, LaRocca et al. ${ }^{[16]}$ concluded that fibrosis originates from the posterior invasion of fibroblasts, extending from erector spinae muscle to the dura and then grows into the hematoma. Songer et al. ${ }^{[17]}$ provided evidence that the adhesion resulted from replacement of hematoma with epidural fat during surgery and that this tissue was then replaced by dense fibrotic tissue. Regardless of the exact mechanism for peridural scar adhesion, it seems to be the consequence of a series of processes involved in wound healing. The imbalance of fibrin deposition and fibrinolysis imposes a high risk of massive peridural adhesion, and hematoma attracts fibrin, which is deposited around the infiltration of the hematoma space.

Holtz et al. ${ }^{[18]}$ proposed a mechanism that could possibly reduce the formation of fibrosis. This involved the following steps: (1) reduction of the initial inflammatory reaction and exudation, (2) inhibition of the coagulated exudates, (3) promotion of removing fibrin, (4) separation of fibrin by a physical barrier, and (5) inhibition of fibro-

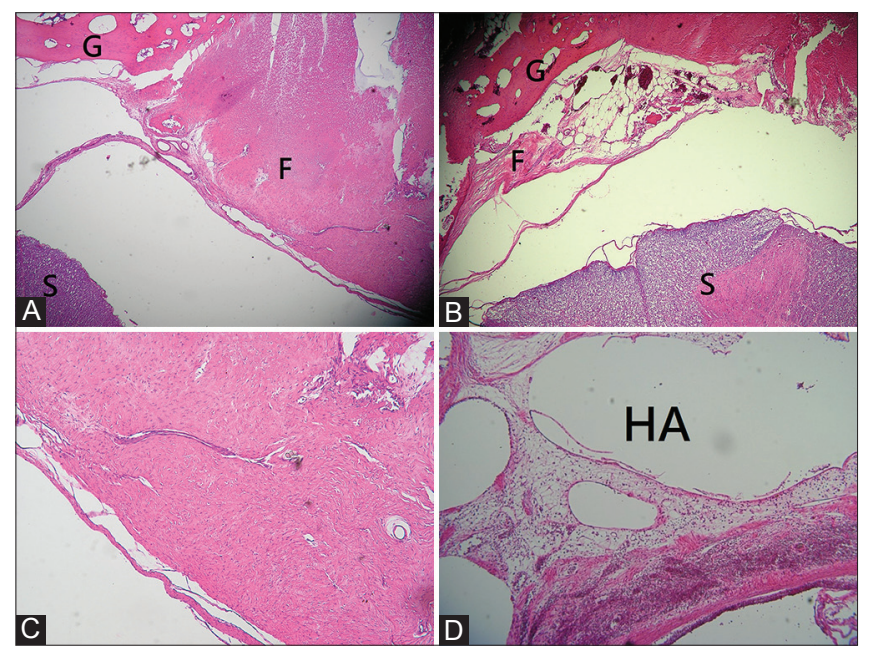

Figure 2: Histologic studies of the laminectomy site indicated significantly less scar tissue formation, a larger subarachnoid space, greater distance from the surface of dura to scar tissues, and fewer inflammatory cells: (A) control group; (B) experimental group; (C) dense fibrous tissue seen in the control group (magnified from a); (D) gelatin-containing vacuolated spaces in the epidural space and surrounded by loose connective tissue in the experimental group (F, fibrous tissue; $\mathrm{G}$, bone tissue; HA, gelatin-containing vacuolated spaces; S, spinal cord). 
blastic proliferation. There are two main ways to reduce initial inflammation: (1) decreasing the tissue injury by using minimally invasive procedure ${ }^{[19]}$ and (2) inhibiting the inflammation pathway by using NSAIDs and steroids; however, this approach can increase the risk of infection. ${ }^{[20]}$

The true relationship between neurologic impairment and peridural fibrosis formation is unclear. It has been estimated that peridural fibrosis or adhesion affects as many as $24 \%$ of patients with failed back surgery syndrome. ${ }^{[2]}$ The fibrotic tissue can restrict the nerve root, limiting its elasticity and thus making it more sensitive to motion, as can be seen during physical examination. Another alternative is that the tissue may occupy the peridural space and make it susceptible to spinal stenosis by ligament hypertrophy or disc bulge. For all these reasons, the amount of scar tissue and its degree of "stickiness" or adhesion should be minimized as much as possible. Most anti-adhesion agents provide a barrier to hematoma by avoiding the contact with the dura mater and nerve root, keeping the fibrotic tissue away from the neurologic organs. An ideal anti-adhesive agent also needs to be effective, biocompatible, and easily applicable, as well as predictably absorbable. ${ }^{[9]}$ Several candidate anti-adhesive materials and methods have been investigated, including low-dose external beam radiation, ${ }^{[5]}$ mechanical barriers such as fat, ${ }^{[21,22]}$ polylactic acid, ${ }^{[23]}$ Avitene $^{\circledR}$ (Davol, Inc., Warwick, RI, USA), and Gelfoam ${ }^{\circledR}$ (Pfizer, Inc., New York, NY, USA), ${ }^{[24]}$ and pharmacological agents such as anti-inflammatory agents, antioxidants, anticoagulants, and fibrinolytics. ${ }^{[25]}$

Results of our study show that HA-based gelatin is an effective and safe anti-adhesion material that can be applied in vivo without any significant adverse effects in an adult rabbit laminectomy model. It has been used as an anti-adhesive material in other anatomic fields, including the intraperitoneal cavity, ${ }^{[25-27]}$ for post-surgery tendon adhesion, ${ }^{[28]}$ and for many years, it has been successfully used for strabismus surgery. ${ }^{[29]}$ HA, a natural extracellular matrix that resides in the human body, has an anti-adhesive effect and acts as a mechanical barrier that separates the injured tissues from the scar tissues by occupying the space and, thus, keeping the hematoma away from the dura mater long enough to have potential benefits for preventing postoperative peridural fibrosis. ${ }^{[30]}$

Adhesion formation is an early phenomenon that occurs primarily within $36 \mathrm{~h}$ of surgery, after hematoma formation. Anti-adhesion barriers must maintain their position on the injured site for at least this amount of time to be effective for preventing adhesion. ${ }^{[31]}$ Hill-West et al.,$^{[32]}$ showed that HA was not effective in the prevention of postsurgical abdominal adhesion; however, their findings were contradicted by the results of other studies. These inconsistent results may be due to the fact that in some cases, the surgical area is greater than the volume of the HA material. In addition, because HA is rapidly absorbed, it may not remain in the peritoneum long enough to form an effective mechanical barrier. As a result, when HA is used as an anti-adhesive agent in abdominal surgery, it is reasonable to consider using it along with a more durable carrier. However, in the case of posterior spinal surgery, the surgical field is more confined to the epidural space and is thus easier to position a barrier from hematoma. As such, half-liquid type HA can infiltrate the surrounding area of the spinal cord and form an adequate space occupied not only as a barrier but also as a tamponade to stop the bleeding. Because of this, an additional carrier for HA may not be needed during spinal surgery.

Fibroblasts may play an important role in the formation of peridural fibrosis originating from the perivertebral muscles and bloodstream. Blocking the migration of fibroblasts to the surgical field is a theoretically applicable measure to prevent peridural fibrosis. However, when Kasimcan et al. ${ }^{[7]}$ evaluated the number of fibroblasts between treatment and control groups, they found no significant difference in the amount of fibroblasts. In our study, there were significantly fewer fibroblasts in the treatment group than in the control group. We hypothesized that since HA is a more biologically inert material, it triggers less inflammatory response than did the bioresorbable material used in Kasimcan et al.'s study. ${ }^{[7]} \mathrm{A}$ more detailed comparison of the effectiveness of HA with other anti-adhesion materials is needed.

HA-based gels have been used as promising anti-adhesive agents in numerous experimental studies. In our study, HA was associated with a reduction of scar tissue, as well as the degree of tenacity between scar tissue and dura mater. HA was absorbed gradually and presented with no adverse effects on local soft tissue healing or on neurologic performance. No potentially serious adverse events were observed in the test animals, and complication rates were similar in the treatment and control groups. Moreover, compared to the control group, the use of HA-based gelatin in the treatment group made no difference in healing of the skin, subcutaneous tissue, or muscle, with one exception: inhibition of peridural fibrosis formation and the adhesion adjacent to the dura at the laminectomy site.

This preliminary study had several limitations. First, the sample size was relatively small. Second, the grading systems for the amount of scar tissue formation, the tenacity between the scar tissue and dura mater, the extent of adhesion, and the scar tissue consistency were relatively subjective, unlike other analyses such as magnetic resonance imaging or quantitative testing, which present more precise images or quantitative results. Third, the tissue samples were harvested at only a single time-point, which is 8 weeks after surgery. The sampling period of anti-adhesive agents in an animal model is usually 4-8 weeks. Anti-adhesion materials are usually absorbed and degraded within 4 weeks. Lalountas et al., ${ }^{[33]}$ and Park et al. ${ }^{[30]}$ compared the effectiveness of different adhesion barriers such as cellulose film and hyaluronan in the prevention of postoperative adhesion 
formation in rats. The samples were harvested and analyzed 2 weeks and 3 weeks after laparotomy. Brzezicki et al., ${ }^{[34]}$ also indicated that a significant difference in the extent of scarring between a laminectomy and sham group could be observed 6 weeks after surgery. Furthermore, Kato et al., ${ }^{[35]}$ demonstrated that it was not possible to quantitatively evaluate the degree of adhesion at 24 weeks after surgery in a rabbit model, because new bone growth was so significant at the laminectomy site in all groups. For all these reasons, we decided to design a study to evaluate the effect of HA in a rabbit laminectomy model, 8 weeks postoperatively. However, future analysis should involve a larger sample and longer observation period. Maturation of scar tissue may occur over a longer period, which might also alter the final results. Finally, the surgical procedure in the current study was limited to laminectomy, excluding discectomy. In the rabbit model, discectomy involves the disruption of annulus fibrosis and the release of material from the nucleus pulposus, which more closely mimics human surgery. It may affect the degree of adhesion and possibly the effectiveness of the anti-adhesion property of HA, which is less reliable in the separation of the anterior aspect of the spinal cord. Furthermore, some authors suggest that some hemostatic characteristics and cytokine inhibitor mixtures can increase scar prevention by decreasing the amount of hematoma and thus lessening the chemotaxis of fibrin. Greater attention to the study design will be needed to develop this product.

Although this was an animal study, it also provides useful information about the effects of hyaluronate in the prevention of postoperative peridural scar adhesion after laminectomy. Nonetheless, further studies should be conducted to determine if HA is harmful to normal tissue, that is, to find whether it results in an increased rate of infection or limits tissue healing. Further clinical trials of the use of HA-based gels should be performed to confirm its effects in human subjects.

\section{Conclusions}

Based on the results of our preliminary study in rabbits, we found that HA-based gelatin is effective for decreasing the tenacity and amount of adhesion between the dura and scar at the laminectomy site. Thus, it can be considered as a potential adhesion barrier for preventing postoperative scar tissue formation.

\section{Acknowledgment}

This work was supported by grant number NSC97-2314-B-182A-009 from the National Science Council of Taiwan.

\section{REFERENCES}

1. North RB, Ewend MG, Lawton MT, Kidd DH, Piantadosi S. Failed back surgery syndrome: 5-year follow-up after spinal cord stimulator implantation. Neurosurgery 1991;28:692-9.

2. Ross JS, Robertson JT, Frederickson RC, Petrie JL, Obuchowski N, Modic MT, et al. Association between peridural scar and recurrent radicular pain after lumbar discectomy: Magnetic resonance evaluation. ADCON-L European Study Group. Neurosurgery 1996;38:855-61-3.

3. Bartynski WS, Petropoulou KA. The MR imaging features and clinical correlates in low back pain-related syndromes. Magn Reson Imaging Clin N Am 2007;15:137-54.

4. Robertson JT. Role of peridural fibrosis in the failed back: A review. Eur Spine J 1996;5 Suppl 1:S2-6.

5. Gerszten PC, Moossy JJ, Bahri S, Kalend A, Martinez AJ. Inhibition of peridural fibrosis after laminectomy using low-dose external beam radiation in a rat model. Neurosurgery. 1999;44:597-602-3.

6. He Y, Revel M, Loty B. A quantitative model of post-laminectomy scar formation. Effects of a nonsteroidal anti-inflammatory drug. Spine (Phila Pa 1976) 1995;20:557-63, 79-80.

7. Kasimcan MO, Bakar B, Aktas S, Alhan A, Yilmaz M. Effectiveness of the biophysical barriers on the peridural fibrosis of a postlaminectomy rat model: An experimental research. Injury 2011;42:778-81.

8. Miyamoto K, Masuda K, Inoue N, Okuma M, Muehleman C, An HS. Anti-adhesion properties of a thrombin-based hemostatic gelatin in a canine laminectomy model: A biomechanical, biochemical, and histologic study. Spine (Phila Pa 1976) 2006;31:E91-7.

9. Shih HN, Fang JF, Chen JH, Yang CL, Chen YH, Sung TH, et al. Reduction in experimental peridural adhesion with the use of a crosslinked hyaluronate/collagen membrane. J Biomed Mater Res B Appl Biomater 2004;71:421-8.

10. Yang J, Ni B, Liu J, Zhu L, Zhou W. Application of liposome-encapsulated hydroxycamptothecin in the prevention of epidural scar formation in New Zealand white rabbits. Spine J 2011;11:218-23.

11. Balazs EA, Denlinger JL. Clinical uses of hyaluronan. Ciba Found Symp 1989;143:265-85.

12. Wu JJ, Shih LY, Hsu HC, Chen TH. The double-blind test of sodium hyaluronate (ARTZ) on osteoarthritis knee. Zhonghua Yi Xue Za Zhi (Taipei) 1997;59:99-106.

13. Einhaus SL, Robertson JT, Dohan FC Jr., Wujek JR, Ahmad S. Reduction of peridural fibrosis after lumbar laminotomy and discectomy in dogs by a resorbable gel (ADCON-L). Spine (Phila Pa 1976) 1997;22:1440-6-7.

14. Moll HD, Schumacher J, Wright JC, Spano JS. Evaluation of sodium carboxymethylcellulose for prevention of experimentally induced abdominal adhesions in ponies. Am J Vet Res 1991;52:88-91.

15. Key JA, Ford LT. Experimental intervertebral-disc lesions. J Bone Joint Surg 1948;30A:621-30.

16. LaRocca H, Macnab I. The laminectomy membrane. Studies in its evolution, characteristics, effects and prophylaxis in dogs. J Bone Joint Surg Br 1974;56B:545-50.

17. Songer MN, Rauschning W, Carson EW, Pandit SM. Analysis of peridural scar formation and its prevention after lumbar laminotomy and discectomy in dogs. Spine (Phila Pa 1976) 1995;20:571-80.

18. Holtz G. Prevention of postoperative adhesions. J Reprod Med 1980;24:141-6.

19. Tullberg T, Rydberg J, Isacsson J. Radiographic changes after lumbar 
discectomy. Sequential enhanced computed tomography in relation to clinical observations. Spine (Phila Pa 1976) 1993;18:843-50.

20. Olmarker K. Reduction of adhesion formation and promotion of wound healing after laminectomy by pharmacological inhibition of pro-inflammatory cytokines: An experimental study in the rat. Eur Spine J 2010;19:2117-21.

21. Gill GG, Sakovich L, Thompson E. Pedicle fat grafts for the prevention of scar formation after laminectomy. An experimental study in dogs. Spine (Phila Pa 1976) 1979;4:176-86.

22. Langenskiold A, Kiviluoto O. Prevention of epidural scar formation after operations on the lumbar spine by means of free fat transplants. A preliminary report. Clin Orthop Relat Res 1976;115:92-5.

23. Mikawa Y, Hamagami H, Shikata J, Higashi S, Yamamuro T, Hyon SH, et al. An experimental study on prevention of postlaminectomy scar formation by the use of new materials. Spine (Phila Pa 1976) 1986;11:843-6.

24. Jacobs RR, McClain O, Neff J. Control of postlaminectomy scar formation: An experimental and clinical study. Spine (Phila Pa 1976) 1980;5:223-9.

25. Belluco C, Meggiolaro F, Pressato D, Pavesio A, Bigon E, Dona M, et al. Prevention of postsurgical adhesions with an autocrosslinked hyaluronan derivative gel. J Surg Res 2001;100:217-21.

26. Urman B, Gomel V, Jetha N. Effect of hyaluronic acid on postoperative intraperitoneal adhesion formation in the rat model. Fertil Steril 1991;56:563-7.

27. Yoldemir T, Sagol S, Adakan S, Oztekin K, Ozsener S, Karadadas N. Comparison of the reduction of postoperative adhesions by two barriers, one solution, and two pharmacologic agents in the rat uterine model. Fertil Steril 2002;78:335-9.
28. Hagberg L, Gerdin B. Sodium hyaluronate as an adjunct in adhesion prevention after flexor tendon surgery in rabbits. J Hand Surg 1992;17:935-41.

29. Yaacobi Y, Hamed LM, Kaul KS, Fanous MM. Reduction of postoperative adhesions secondary to strabismus surgery in rabbits. Ophthalmic Surg 1992;23:123-8.

30. Park JS, Cha SJ, Kim BG, Choi YS, Kwon GY, Kang H, et al. An assessment of the effects of a hyaluronan-based solution on reduction of postsurgical adhesion formation in rats: A comparative study of hyaluronan-based solution and two film barriers. J Surg Res 2011;168:49-55.

31. Harris ES, Morgan RF, Rodeheaver GT. Analysis of the kinetics of peritoneal adhesion formation in the rat and evaluation of potential antiadhesive agents. Surgery 1995;117:663-9.

32. Hill-West JL, Chowdhury SM, Dunn RC, Hubbell JA. Efficacy of a resorbable hydrogel barrier, oxidized regenerated cellulose, and hyaluronic acid in the prevention of ovarian adhesions in a rabbit model. Fertil Steril 1994;62:630-4.

33. Lalountas M, Ballas KD, Michalakis A, Psarras K, Asteriou C, Giakoustidis DE, et al. Postoperative adhesion prevention using a statin-containing cellulose film in an experimental model. Br J Surg 2012;99:423-9.

34. Brzezicki G, Jankowski R, Blok T, Klimczak A, Szymas J, Huber J, et al. Postlaminectomy osteopontin expression and associated neurophysiological findings in rat peridural scar models. Spine (Phila Pa 1976) 2011;36:378-85.

35. Kato T, Haro H, Komori H, Shinomiya K. Evaluation of hyaluronic acid sheet for the prevention of postlaminectomy adhesions. Spine J 2005;5:479-88. 